\title{
The Stellar and Gas Kinematics of Several Irregular Galaxies
}

\author{
Deidre A. Hunter ${ }^{1}$ \\ Lowell Observatory, 1400 West Mars Hill Road, Flagstaff, Arizona 86001 USA \\ dah@lowell .edu \\ Vera C. Rubin ${ }^{1}$ \\ Carnegie Institution of Washington, 5241 Broad Branch Road, NW, Washington, D. C. \\ 20015 USA \\ rubin@dtm.ciw.edu \\ Rob A. Swaters \\ Department of Physics and Astronomy, Johns Hopkins University, 3400 N. Charles Street \\ and Space Telescope Science Institute, 3700 San Martin Dr., Baltimore, MD 21218 \\ swaters@pha.jhu.edu \\ Linda S. Sparke \\ Washburn Observatory, 475 North Charter Street, Madison, WI 53706-1582 USA \\ sparke@astro.wisc.edu \\ and \\ Stephen E. Levine \\ US Naval Observatory, Flagstaff Station, PO Box 1149, Flagstaff, AZ 86002-1149 USA \\ sel@nofs.navy.mil
}

\begin{abstract}
${ }^{1}$ Visiting Astronomer, Kitt Peak National Observatory, National Optical Astronomy Observatory, which is operated by the Association of Universities for Research in Astronomy, Inc. (AURA) under cooperative agreement with the National Science Foundation.
\end{abstract}


We present long-slit spectra of three irregular galaxies from which we determine the stellar kinematics in two of the galaxies (NGC 1156 and NGC 4449) and ionized-gas kinematics in all three (including NGC 2366). We compare this to the optical morphology and to the HI kinematics of the galaxies. In the ionized gas, we see a linear velocity gradient in all three galaxies.

In NGC 1156 we also detect a weak linear velocity gradient in the stars of $(5 \pm 1 / \sin i) \mathrm{km} \mathrm{s}^{-1} \mathrm{kpc}^{-1}$ to a radius of $1.6 \mathrm{kpc}$. The stars and gas are rotating about the same axis, but this is different from the major axis of the stellar bar which dominates the optical light of the galaxy.

In NGC 4449 we do not detect organized rotation of the stars and place an upper limit of $(3 / \sin i) \mathrm{km} \mathrm{s}^{-1} \mathrm{kpc}^{-1}$ to a radius of $1.2 \mathrm{kpc}$. For NGC 4449, which has signs of a past interaction with another galaxy, we develop a model to fit the observed kinematics of the stars and gas. In this model the stellar component is in a rotating disk seen nearly face-on while the gas is in a tilted disk with orbits whose planes precess in the gravitational potential. This model reproduces the apparent counter-rotation of the inner gas of the galaxy. The peculiar orbits of the gas are presumed due to acquisition of gas in the past interaction.

Subject headings: galaxies: irregular — galaxies: kinematics and dynamics galaxies: structure - galaxies: individual (NGC 1156, NGC 2366, NGC 4449)

\section{Introduction}

We have learned that irregular galaxies are not just small versions of spiral disks, but we do not know the intrinsic characteristics of these systems, the most common type of galaxy in the universe. Indeed, the fundamental structure of irregular galaxies is still in doubt. It has been generally believed that irregular galaxies are disks like spirals, and like spirals, surface brightness profiles in most irregulars are most often adequately fit with an exponential law (Patterson \& Thuan 1996; van Zee 2000). However, studies of the distributions of projected minor-to-major axis ratios $b / a$ suggest that irregulars are thicker than spirals, perhaps having intrinsic flattening ratios $(b / a)_{0}$ of $0.3-0.4$ rather than the 0.2 value generally adopted for spirals (Hodge \& Hitchcock 1966, van den Bergh 1988, Binggeli \& Popescu 1995). Staveley-Smith, Davies, \& Kinman (1992), on the other hand, argue that the low ratio of rotation velocity to velocity dispersion often seen in irregulars must imply a thick disk with an intrinsic flattening ratio as high as 0.6. More recently Sung et al. (1998) have also analyzed ellipticity distributions and concluded that dwarf irregulars and Blue Compact Dwarfs (BCDs) are more triaxial than disk-shaped, having axis ratios 1:0.7:0.5 
and being only a little less spherical than dwarf ellipticals. What then is the true shape of irregulars?

Because of these outstanding issues, we have begun a program aimed at understanding the intrinsic structure of normal irregular galaxies through determining their stellar kinematics. In this paper we present results from long-slit spectral observations of stellar absorption features in two irregular galaxies of high surface brightness. While observations of giant galaxies are often detailed enough to justify sophisticated analysis of their structure, (see, for example, Statler \& Fry 1994; Statler, Smecker-Hane, \& Cecil 1996; Statler \& SmeckerHane 1999), observations of the stellar kinematics of irregular galaxies have been harder to obtain (but see Swaters 1999). Most irregular galaxies are much lower in surface brightness than giant galaxies, and they rotate much more slowly, so a higher velocity resolution is needed. Thus, the signal-to-noise that is possible with current instrumentation limits what can be deduced for irregular galaxies. Nevertheless, we can begin to learn about the highest surface brightness irregulars now, and expect to extend to lower surface brightness objects as long-slit spectrographs or field integrators with moderate spectral resolution become available on very large telescopes.

An understanding of the shape of Im galaxies is important to understanding other aspects of these systems. A colleague of ours saw a broad-band optical picture of the irregular galaxy Sextans A (Hunter 1997) on a bulletin board and asked why the galaxy appears square and has such sharp edges. Sextans A is not alone; other irregular galaxies also defy our expectations of what galaxies should look like. The rectangular shape of these galaxies is often taken to mean that the galaxies are barred. But, if that is the case, their bar structures are very different from those in spirals. In spirals the bar length is typically $\leq 0.3$ of the size of the stellar disk (Elmegreen \& Elmegreen 1985). In the rectangular irregulars the bar is a significant fraction of the entire optical galaxy. For example, in NGC 4449 the bar has a length that is $90 \%$ of $\mathrm{D}_{25}$, the diameter measured to a B-band surface brightness level of 25 magnitudes $\operatorname{arcsec}^{-2}$ (Hunter, van Woerden, \& Gallagher 1999). What does it mean for most of the galaxy to be a bar?

There are also often peculiarities in the gas kinematics and distributions that are not evident in the optical morphology. In Sextans A the velocity field of the neutral gas is not aligned with the optical axis of symmetry and there is some evidence that gas is moving on elliptical orbits (Skillman et al. 1988). In NGC 4449 the inner gas is counter-rotating with respect to the outer gas and neither gas system is aligned with the isophotes of the optical galaxy (Hunter et al. 1999). However, because of its large cross-section, the gas of many irregulars could have been perturbed by outside forces over the galaxy's lifetime (for example, NGC 4449: Hunter et al. 1998). What are the kinematics of the stellar system, 
which has a smaller cross-section, and how do they compare to that of the gas?

In this paper we report results of our first observations of stellar kinematics determined from absorption spectra in the irregulars NGC 1156 and NGC 4449. We also discuss the kinematics of the ionized gas in these two galaxies as well as in NGC 2366.

\section{The Galaxy Sample}

Two of the galaxies in our sample are high surface brightness irregular galaxies: NGC 1156 and NGC 4449. They have $\mu_{25}$, the average surface brightness within $\mathrm{D}_{25}$, of 22.4 and 22.1, respectively. The third galaxy, NGC 2366, is a more typical, lower surface brightness irregular $\left(\mu_{25} \sim 23.4\right)$. All three galaxies are classified as IBm, barred Magellanic-type irregulars, by de Vaucouleurs et al. (1991; 三RC3). Only NGC 2366 might be considered a "dwarf" irregular under any of the more common luminosity-based definitions adopted by authors. In Table 1 we list some global properties of the galaxies that are pertinent to our discussion. Figure 1 shows $\mathrm{V}$-band and $\mathrm{H} \alpha$ images of each galaxy.

At an $\mathrm{M}_{B}$ of -18.0 , NGC 1156 is at the high end of the range of luminosities seen in normal irregular galaxies (Hunter 1997), and is about 25\% brighter than the Large Magellanic Cloud in the B-band. NGC 1156 has a modestly high rate of star formation and H II regions are crowded over the disk. Karachentsev, Musella, \& Grimaldi (1996) describe NGC 1156 as "the less [sic] disturbed galaxy in the Local Universe," and there are no significant galaxy neighbors within $0.7 \mathrm{Mpc}$ and $\pm 500 \mathrm{~km} \mathrm{~s}^{-1}$. However, in HI channel maps McIntyre (2002) sees a structure that resembles a tiny tidal arm. It terminates at the northeast HI complex, where there is an unusually large range in velocity (Swaters 1999), and extends $3^{\prime}$ to the southeast. Although no other evidence of companions is seen, it is possible that NGC 1156 is not the pristine galaxy that it once seemed.

NGC 4449 is one of the most luminous and actively star-forming IBm galaxies (RC3, Hunter 1997). It has an integrated $\mathrm{M}_{B}$ of -18.2 , making it 1.4 times as luminous as the LMC. It is forming stars twice as rapidly as the LMC even though the sizes of these two galaxies are similar. NGC 4449 is also unusual in having neutral hydrogen gas at $10^{19}$ atoms $\mathrm{cm}^{-2}$ extending to six times its Holmberg radius (van Woerden, Bosma, \& Mebold 1975; Bajaja, Huchtmeier, \& Klein 1994), a distribution that is about three times more extended than that of most other irregular galaxies (Huchtmeier, Seiradakis, \& Materne 1981). Van Woerden et al. (1975) and Bajaja et al. (1994) found opposite velocity gradients between gas in the inner few arcminutes and the gas beyond that. Hunter et al. (1998) resolved part of the extended gas into enormous streamers that wrap around the galaxy. These, and 
the counter-rotating gas systems, suggest that NGC 4449 has undergone an interaction and possibly a merger with another galaxy (Hunter et al. 1998). NGC 4449 is a member of the CVnI "loose" cloud of galaxies (de Vaucouleurs 1975), and the small irregular DDO 125 is only $41 \mathrm{kpc}$ away in the plane of the sky. Models by Theis \& Kohle (2001) suggest that DDO 125 was the interaction partner that produced the streamers of gas that we now see around NGC 4449, but their models do not address the counter-rotating gas system in the central part of the galaxy.

NGC 2366, located in the M81 Group of galaxies, is a lower luminosity irregular, having almost the same absolute B magnitude as the Small Magellanic Cloud. It contains the supergiant H II complex NGC 2363 to the southwest of the galaxy center. NGC 2363 is nearly twice as bright in $\mathrm{H} \alpha$ as the 30 Doradus nebula in the Large Magellanic Cloud and contains a large fraction of the total current star-formation activity of the galaxy (Aparicio et al. 1995, Drissen et al. 2000). However, there is also another large H II complex to the west of NGC 2363 and numerous smaller H II regions scattered along the disk.

\section{The Spectroscopic Observations, Data Reduction, and Analysis}

\subsection{Long-slit spectra}

We obtained long-slit spectra of our three galaxies during a 4-night observing run in 2000 January at the Kitt Peak National Observatory (KPNO) $4 \mathrm{~m}$ telescope. We used the RC-spectrograph and a Tektronics $2048 \times 2048$ CCD detector. The detector gain was $3.1 \mathrm{e}^{-}$ $\mathrm{ADU}^{-1}$, and the readnoise was $4.0 \mathrm{e}^{-}$. We used a $3^{\prime \prime}$-wide slit without a decker in order to obtain the longest slit possible. The useful portion of the slit was 463 pixels $\left(5.5^{\prime}\right)$ in length, and from a series of star observations we determined the scale to be $0.71^{\prime \prime}$ pixel $^{-1}$. The stellar FWHM (along the slit) was $\sim 2.5^{\prime \prime}$ near the center of the spectra. The focus degraded both blueward and redward of that so that at $4840 \AA$ the FWHM of a star was $6^{\prime \prime}$ and the profile became double-peaked with a central minimum; at $5274 \AA$ the FWHM was $3.1^{\prime \prime}$.

With grating BL380 in second order and order-separating filter BG39 we covered 4800$5350 \AA$ at a dispersion of $0.36 \AA$ pixel $^{-1}$. The spectral resolution was $2.0-2.5$ pixels, measured from comparison lamp emission lines and night sky lines. A typical value of 2.2 pixels corresponds to $47 \mathrm{~km} \mathrm{~s}^{-1}$ at $[\mathrm{OIII}] \lambda 5007$. We chose this particular wavelength region in order to include the MgIb absorption features at 5183.6 $\AA$, $5172.7 \AA$, and $5167.3 \AA$. These features are stronger in the cooler stars (late $\mathrm{F}$ through $\mathrm{M}$ ). However, the cross-correlation technique includes all absorption lines detected in the spectra, not just the MgIb features. In addition to the absorption lines, our spectra included emission lines, the strongest of which 
are $\mathrm{H} \beta$ and $[\mathrm{OIII}] \lambda 4959,5007$. These emission lines allow us to obtain the radial velocities of the ionized gas for comparison to that of the stars.

We used an FeAr comparison lamp for wavelength calibration, and took a calibration observation before and after each object spectrum. The electronic pedestal was subtracted using the overscan strip of each image and separate bias frames to remove two-dimensional structure. Pixel-to-pixel variations were removed using observations of a white screen hanging on the inside of the dome. We also obtained twilight sky flats to determine the slit illumination function, but this was not an important correction for our purposes and it was applied only to the version of the spectra with linear wavelength repixelization. We observed a star stepped along the slit in order to remove S-distortion, but found that the amount of distortion was small compared to errors in fitting the centers of the stellar spectra and so did not apply this correction. During the run we observed radial velocity standard stars from the United States Nautical Almanac Office (2000) and other late-type stars chosen from Jacoby, Hunter, \& Christian (1984) to use as templates in the cross-correlation. The template stars that we used are listed in Table 2. We also used the twilight sky as the template of a G2V star. There was thin cirrus during a few observations, especially on the first night, but skies were clear otherwise.

We observed NGC 1156 and NGC 4449 at a different position angle (PA) each of the four nights of the observing run. We oriented the slit along the optical major axis determined from a deep V-band image, along the minor axis, and at plus and minus $45^{\circ}$ from the major axis. Note that we define the position along the slit measured from the center of the galaxy in the direction of the given position angle as positive, and positions along the slit in the opposite direction as negative. Each slit was placed on the galaxy using a center predetermined from the V-band images, as given in Table 1 . We offset to these positions from nearby stars. One exception was NGC 1156's spectrum at PA $174^{\circ}$ which was offset $2^{\prime \prime}$ east of the original position in order to avoid a bright star cluster. At each PA we observed a series of 2700 s exposures: 5-6 each of NGC 1156 and 4 each of NGC 4449. The object spectra were preceded, interleaved, and followed with comparison lamp observations. Because NGC 4449 is so large, observations of it were also preceded, interleaved, and followed with separate sky observations taken $1^{\circ}$ (nights 1 and 2) or $6^{\prime}$ (nights 3 and 4) alternately north and south of the galaxy.

Because NGC 2366 is much lower in surface brightness than NGC 1156 and NGC 4449, we did not expect to detect absorption features well enough to measure stellar kinematics in this galaxy in the available time. Therefore, we only observed this galaxy for the emissionline kinematics. We obtained observations at 4 different PA: Three were placed on the center of the galaxy determined from our V-band image; the fourth at PA $27^{\circ}$ was centered at $7^{\mathrm{h}}$ 
$29^{\mathrm{m}} 02.5^{\mathrm{s}}, 69^{\circ} 14^{\prime} 17^{\prime \prime}(2000)$ in order to reach the nebulae in the outer part of the galaxy. Exposures at PAs $30^{\circ}$ and $120^{\circ}$ were $3600 \mathrm{~s}$ each; spectra at PAs $63^{\circ}$ and $27^{\circ}$ were single $1800 \mathrm{~s}$ exposures. A separate sky observation was obtained only for one of the observations at PA $30^{\circ}$.

After the spectra were corrected for the electronic pedestal and pixel-to-pixel variations, we did a two-dimensional fit to each of the comparison lamp spectra. We then performed a two-dimensional transformation of each object frame using the nearest comparison. If an object frame was bracketed by comparisons, we did the two-dimensional fit to both comparisons simultaneously, effectively averaging any wavelength shifts. On one of the nights, moving a mirror into place for observations of the comparison lamp generated an electronic signal that moved the grating by two encoder units ( 6 pixels). The motion was discrete in time, and therefore, the observations of the comparison lamp were readily used to remove this shift. Our transformation repixelizes in the dispersion direction in order to impose a uniform wavelength scale, and it corrects for curvature along lines of constant wavelength. We did the transformations both to a linear wavelength scale and to a logarithmic scale; the linear versions were used to measure the emission-line radial velocities and the logarithmic versions were used to measure the absorption velocities using cross-correlation techniques.

We checked that the final galaxy spectra were aligned in wavelength by overplotting night sky lines. When multiple spectra were obtained for a given PA, we combined them with a rejection algorithm to remove cosmic rays. For NGC 2366 where we had single exposures, we edited cosmic rays by hand. For NGC 1156, the background was removed using the region of the slit beyond the galaxy. The background from NGC 2366 spectra were removed in this way as well for all but one of the two observations at $\mathrm{PA} 30^{\circ}$ for which a separate sky observation was available. For NGC 4449, separate sky frames were combined and subtracted from object frames. For the stars, we fit the background on either side of the star profile.

\subsection{Cross-correlation and absorption velocities}

To determine the radial velocity of the stars from absorption lines, we used a crosscorrelation technique that matches absorption lines of template stars with the object spectrum (Simkin 1974; Tonry \& Davis 1979; Statler 1994). We began by extracting the onedimensional template star spectra from the two-dimensional spectra by summing along the slit to where the starlight blended into the background. For the object spectra, we wanted to increase the signal-to-noise as much as possible but without spatially summing over regions corresponding to large changes in radial velocity. Therefore, we extracted one-dimensional 
object spectra by summing every 5,15 , and 25 rows $\left(3.55^{\prime \prime}, 10.65^{\prime \prime}, 17.75^{\prime \prime}\right)$ and comparing the results for the different summing intervals. We finally adopted the results for the 15-row sums, and those measurements are what are presented here. Spectral regions with emission lines were excluded in the cross-correlation.

Each object spectrum was compared with each F-K-type template star, giving an estimate of the heliocentric radial velocity for each fit. We averaged these velocities, weighted by the formal uncertainties of the fit to the cross-correlation peak. The uncertainties of each cross-correlation are of order 5-20 $\mathrm{km} \mathrm{s}^{-1}$, depending on the signal-to-noise. There were no systematics greater than $0.3 \mathrm{~km} \mathrm{~s}^{-1}$ involving any particular template star. On the one night that we did two radial velocity standards, cross-correlation of one with the other reproduces the $\mathrm{V}_{\text {helio }}$ to $2.5 \mathrm{~km} \mathrm{~s}^{-1}$, and this is probably a good measure of the uncertainties for high signal-to-noise spectra and a lower limit on the uncertainty of the galaxy measurements.

DAH and RAS independently applied the cross-correlation technique to the galaxy data, using different approaches to subtracting the continuum and fitting the cross-correlation peaks. Comparing our results gave us a good feeling for the real uncertainties in our results. For all but a few points the agreement was within a few $\mathrm{km} \mathrm{s}^{-1}$, well within the formal uncertainties. However, in NGC 1156 the point at $\mathrm{r}=-42^{\prime \prime}$ in PA $129^{\circ}$ differed by $24 \mathrm{~km}$ $\mathrm{s}^{-1}$, the point at $\mathrm{r}=-52^{\prime \prime}$ in PA $84^{\circ}$ differed by $18 \mathrm{~km} \mathrm{~s}^{-1}$, and the point at $\mathrm{r}=-21^{\prime \prime}$ and $-31^{\prime \prime}$ in PA $174^{\circ}$ differed by $11 \mathrm{~km} \mathrm{~s}^{-1}$. These differences are, nevertheless, within $1-2 \sigma$. In addition to the two cross-correlation approaches, VCR used the technique described below for measuring emission lines to measure the centroid of the MgIb absorption lines in the central parts of two of the NGC 1156 spectra. This is a completely different approach from cross-correlation and involves inverting the spectrum to make the absorption lines look like emission lines. Those measurements agreed well with the results of the cross-correlation technique, although it could only be applied to the brighter regions of the spectra. All of these experiments gave us confidence in our absorption line velocity measurements and their uncertainties.

We used the radial velocity standards as template stars and cross-correlated the absorption lines to determine the radial velocities of the other template stars. To further fine tune the zero points, we determined the central velocity $\mathrm{V}_{\text {sys }}$ of the galaxy in each spectrum where that was reliable to do and took the average. The radial velocities from each spectrum were then adjusted to match this $\mathrm{V}_{\text {sys }}$. The measured and average $\mathrm{V}_{\text {sys }}$ and amount $\Delta \mathrm{V}_{\text {sys }}$ by which the velocities measured from each spectrum were adjusted are given in Table 3 . 


\subsection{Emission-line measurements}

The emission spectra were measured by VCR using a customized measuring program in the software package VISTA (Rubin, Hunter, \& Ford 1991). The program fits a 2-dimensional polynomial to lines across a spectrum, and then returns the wavelength and radial position for each pixel; no repixelling is done.

For the current observations, several very minor modifications were made. First, the comparison FeAr frames for each PA were summed, using a weighting of 1 for a comparison taken between exposures, and a weight of 0.5 for a comparison taken at the beginning or end of each set. This assured that the individual comparison frames were handled in the same manner as the galaxy frames. The (rectified, summed) comparison was then fit with a low order polynomial whose coefficients were transfered to the corresponding galaxy frame. For each of the 12 summed comparison frames, the fit to 21 lines (4806 $\AA-5328 \AA$ ), each sampled at 16 radial positions, had an rms of $0.04 \AA$, equivalent to $2.4 \mathrm{~km} \mathrm{~s}^{-1}$. We are satisfied that we have preserved the velocity and radial scales from the comparison arcs to this level. Even though preliminary tests had shown that velocities differed only by a few $\mathrm{km}$ $\mathrm{s}^{-1}$ in measuring the summed galaxy spectrum with any one of the bracketing comparisons, we preferred to sum the comparison frames so as to treat them in a manner analogous to that used for the galaxy spectra.

Only the measurements of the $[\mathrm{OIII}] \lambda 5007$ line are used in the analysis that follows. $\mathrm{H} \beta$ emission is superposed on a broad absorption line, and [OIII] $\lambda 4959$ is only one-third as strong as $\lambda 5007$, causing their measures to have lower weight. In addition, the focus of the spectra degrades below about $4950 \AA$, causing the emission lines to develop a faint red wing. We have confirmed that this does not affect the $\lambda 5007$ emission line, and that there is no correlation between line intensity and velocity. We did measure both $\lambda 4959$ and $\lambda 5007$ for a few spectra and found that they were the same to within a few $\mathrm{km} \mathrm{s}^{-1}$. We adopt an intrinsic wavelength of $5006.853 \AA$ for [OIII] (Kaufman \& Sugar 1986).

The measuring program fits a Gaussian to the emission in a given line at successive points along the slit, and the intensity-weighted centroid of the measures is adopted as the velocity there. Tests have shown that the centroid is a more stable measure than the peak. In regions where the emission is strong, a measurement is taken at each pixel $\left(0.71^{\prime \prime}\right)$ in the radial direction. Where the emission is weak, measurements are integrated over 5 or 6 pixels $\left(3.5^{\prime \prime}-4.3^{\prime \prime}\right)$. The accuracy of each radial velocity is about $\pm 5 \mathrm{~km} \mathrm{~s}^{-1}$ on the plane of the sky. 


\subsection{Stellar velocity dispersion}

Fitting of the cross-correlations peaks also yields a FWHM of the profile, which is a measure of the velocity dispersion. The resolution of the cross-correlation fitting is of order $70 \mathrm{~km} \mathrm{~s}^{-1}$ (square-root of 2 times the spectral resolution). For NGC 1156 the FWHM of the cross-correlation profiles are not resolved. For NGC 4449, the profile may be marginally resolved, indicating a high stellar velocity dispersion, but the results depend strongly on the template star used. The velocity dispersion in the neutral gas is also unusually high in this galaxy: of order $20 \mathrm{~km} \mathrm{~s}^{-1}$ within the optical galaxy rather than the usual $10 \mathrm{~km}$ $\mathrm{s}^{-1}$ seen in most gas disks (Hunter et al. 1999). However, given the low resolution and the uncertainties resulting from the choice of template star, we do not feel confident in stating a stellar velocity dispersion from these data for either galaxy. The velocity dispersion has been measured in the Sm galaxy UGC 4325 (=NGC 2552) by Swaters (1999) and found to be $19 \pm 2 \mathrm{~km} \mathrm{~s}^{-1}$. A velocity dispersion this small is not measureable with our data.

\section{Results: NGC 2366}

One spectrum was obtained with the slit placed at a $\mathrm{PA}$ of $30^{\circ}$, along the major axis as shown in Figure 1. One can see that this PA fairly respresents the major axis of the galaxy at all surface brightness levels (see Hunter, Elmegreen, \& van Woerden [2001] for more discussion). Another spectrum was obtained with the slit placed along the morphological minor axis of $120^{\circ}$, and one at a PA of $63^{\circ}$ was designed to pick up the supergiant HII region to the southwest. These three spectra were obtained with the slits placed at the center of the galaxy. The fourth spectrum at $\mathrm{PA}=27^{\circ}$ is displaced $113^{\prime \prime}$ from the center along $\mathrm{PA}=27^{\circ}$ in order to intersect the prominent nebulosity to the north. The four slit positions are shown superposed on an $\mathrm{H} \alpha$ image of the galaxy in Figure 1.

Strong ionized-gas emission lines of $\mathrm{H} \beta$ and [OIII] $\lambda 5007$ and $\lambda 4959$ are observed in all PAs. Weak lines of Fe I (4986 $\AA, 5015 \AA)$ are detected from the large southwestern emission region NGC 2363 and from the northeastmost knot in $\mathrm{PA}=63^{\circ}$. In every case, these weaker lines arise in the regions with the strongest emission at $\mathrm{H} \beta$ and $[\mathrm{OIII}] \lambda 4959,5007$. In $\mathrm{PA}=30^{\circ}$ only, an emission line is seen at $5096 \AA$ arising in a region of relatively strong continuum but only moderate line emission and extending into a region of low continuum. This line emission comes from the region northeast of the circular ring, about $50^{\prime \prime}-67^{\prime \prime}$ from the adopted center. In other knots with even stronger continua, this emission line is not observed.

We plot $\mathrm{V}_{\text {helio }}$ measured in the plane of the sky from each emission spectrum in Figure 2. Linear rotation is observed generally northeast of the center in PA $27^{\circ}, 30^{\circ}$, and $63^{\circ}$ 
to $1.9 \mathrm{kpc}$. In $\mathrm{PA}=120^{\circ}$, the morphological minor axis, velocities are essentially flat, with peak-to-peak velocity variations only of order $\pm 10 \mathrm{~km} \mathrm{~s}^{-1}$. This would imply a kinematical minor axis of $\mathrm{PA}=120^{\circ}$, and major axis of about $30^{\circ}$.

The ionized gas in NGC 2366 exhibits deviations from overall rotation due to the energetic output of massive stars in star-forming regions. There are two supergiant H II regions in the southwest part of the galaxy - NGC 2363 and NGC 2366-III - that are known to exhibit high kinetic energies in the ionized gas (Hunter 1982; Roy et al. 1992; Hunter \& Gallagher 1997; Martin 1997). We see this at negative RA at PAs of $30^{\circ}$ and $63^{\circ}$ in Figure 2.

The ionized gas and HI have similar rotation velocities in the inner part of the galaxy. This can be seen in Figure 3 where the velocities of the ionized gas are superposed on position-velocity diagrams made from slices through the HI data (Hunter et al. 2001). We see that the ionized gas velocities nearly follow the ridge-line of the neutral gas with mostly modest deviations of the ionized gas from HI peaks. Tomita et al. (1998) found differences between the velocities of $\mathrm{H} \alpha$ and $\mathrm{HI}$ in NGC 2366 of order $\pm 10 \mathrm{~km} \mathrm{~s}^{-1}$, consistent with what we see.

Major axis PAs have been measured from optical surface photometry and from the HI velocity field as well. From R-band surface photometry, Swaters (1999) determined a PA of $28^{\circ}$ for the major axis of NGC 2366. Hunter et al. (2001) used a V-band image and found a PA of $32^{\circ}$; the exponential fit deduced from the $\mathrm{V}$-band surface photometry to $7.5^{\prime}$ radius also fit well the J-band surface photometry as far as it was measured ( $4^{\prime}$ radius). These agree well with the nominal PA of $30^{\circ}$ for the emission-line kinematics seen here.

Modeling of the HI velocity field, on the other hand, produces different parameters. The $21 \mathrm{~cm}$ observations do indicate an overall rotation, but with a notable asymmetry at large radii $\left(>2^{\prime}\right)$. Modeling of $21 \mathrm{~cm}$ kinematic data over the galaxy as a whole gives a major axis of $39^{\circ}-46^{\circ}$ (Braun 1995; Wevers, van der Kruit, \& Allen 1986; Swaters 1999; Hunter et al. 2001). The inclination angles infered from the HI velocity field modeling are also different from those determined from the optical surface photometry, being smaller by $7^{\circ}-14^{\circ}$. These differences suggest that the HI distribution or kinematics deviate from axisymmetry; that is, there are noncircular motions in the HI or the HI disk is warped.

We have examined several models to describe the optical emission-line kinematics of the inner $\sim 2^{\prime}$ radius: 1 ) major axis at $\mathrm{PA}=30^{\circ}$ (near that determined from the optical surface photometry), and 2) major axis at $\mathrm{PA}=45^{\circ}$ (near that determined from the HI kinematics). Both models assume that the galaxy is circular in its principal plane, that the adopted center is the center of mass, and that internal motions in the emission regions are small with respect to the rotation velocities. For a galaxy with small rotation velocity, this latter assumption 
is likely to be violated in the strong emission complexes such as we see in the southwest portion of NGC 2366.

Model 1: We adopt $30^{\circ}$ as the PA of the stellar kinematical major axis. A weighted leastsquares fit to the line-of-sight velocities, $+110^{\prime \prime}<\mathrm{r}<-46^{\prime \prime}$, gives a gradient of $(0.50 \pm 0.04 / \sin i)$ $\mathrm{km} \mathrm{s}^{-1} \operatorname{arcsec}^{-1}$ or $(30.4 \pm 2.2 / \sin i) \mathrm{km} \mathrm{s}^{-1} \mathrm{kpc}^{-1}$ (solid line in Fig. 2). To the southwest at larger distances from the center, the optical velocities are flat at about $80 \mathrm{~km} \mathrm{~s}^{-1}$. For an inclination of $72^{\circ}$ determined from optical surface photometry, under the assumption that these are thick disks with intrinsic $(b / a)_{0}$ of 0.3 (Hodge \& Hitchcock 1966; van den Bergh 1988), the gradient in the plane of the galaxy is $32 \pm 2 \mathrm{~km} \mathrm{~s}^{-1} \mathrm{kpc}^{-1}$. This rotation curve, deprojected to $\mathrm{PA}=63^{\circ}$ and overplotted on the $\mathrm{PA}=63^{\circ}$ measured velocities (Fig. 2), is a moderately good representation of the observed velocities to the north; but not to those in the south where non-circular motions dominate.

Model 2: For this model, we adopt the $\mathrm{PA}$ of the major axis as $\approx 45^{\circ}$, the approximate mid-point of $27^{\circ}, 30^{\circ}$ and $63^{\circ}$. Hence, in all three PAs, velocities should be similar. We show in Figure 4 line-of-sight velocities measured in the region $+125^{\prime \prime}<\mathrm{r}<-50^{\prime \prime}$ for these three PAs. Their similarity over this region is striking. A weighted least-squares fit produces a slope of $0.50 \pm 0.02 \mathrm{~km} \mathrm{~s}^{-1} \operatorname{arcsec}^{-1}$ (solid line in Fig. 4), the same as that for Model 1, above. Along the adopted major axis, $\mathrm{PA}=45^{\circ}$, the rotation curve would have a velocity gradient of $(0.52 \pm 0.02 / \sin i) \mathrm{km} \mathrm{s}^{-1} \operatorname{arcsec}^{-1}$ or $(31 \pm 1 / \sin i) \mathrm{km} \mathrm{s}^{-1} \mathrm{kpc}^{-1}$. This rotation curve, projected onto the four PAs in the plane of the sky are shown in Figure 2 (dashed line); the radial extent of the rotation curve deprojects to $\mathrm{r}>-20^{\prime \prime}$ along what is a near-minor axis in $\mathrm{PA}=120^{\circ}$ in this model. For $i=72^{\circ}$, the gradient is $33 \pm 1 \mathrm{~km} \mathrm{~s}^{-1} \mathrm{kpc}^{-1}$.

There is little reason from these data for choosing between these models. Both fit the northeast gradients along the three position angles. Although the minor axis velocities are reproduced by both models, the observed small velocity gradient at $\mathrm{r}>-20^{\prime \prime}$ in $\mathrm{PA}=120^{\circ}$ offers some evidence that a major axis near $45^{\circ}$ is a slightly better fit. For any model, the lack of observed rotation for $\mathrm{r}<-20^{\prime \prime}$ on the southwest side is assumed to be due to NGC 2363 and HII region NGC 2366-III whose internal motions distort the systemic velocity pattern. However, observations by Odewahn (1989) suggest that there may also be a twisting of the isovelocity contours in this region that implies a more systematic deviation from the velocity field in the rest of the galaxy.

The resulting rotation curves for NGC 2366 are shown in Figure 5. Superposed on this is the rotation curve derived from the HI (Hunter et al. 2001). There is fair agreement between the optical and the $21 \mathrm{~cm}$ curves in the regions where they overlap. However, the nuclear rise of the optical velocities is steeper and the linear rotation extends further in the ionized gas than in the HI. This is the result of the differences in resolution of the two data; 
the FWHM of the HI beam is $34^{\prime \prime} \times 29^{\prime \prime}$ while the resolution of the optical spectra is a few arcseconds. Points at large distance from the center of the galaxy, however, are consistent with the leveling-off in the rotation curve seen in the HI.

\section{Results: NGC 1156}

A deep V-band image of NGC 1156 reveals a rectangular shape that grows more circular at low surface brightness levels. The logarithm of the V-band image is shown in Figure 1 in order to allow comparison of the morphology of the galaxy at different surface brightness levels. We had used this $\mathrm{V}$-band image to determine the morphological major axis of the galaxy, 39 ${ }^{\circ}$. A line is drawn along this axis in Figure 1. From R-band surface photometry Swaters (1999) determined the major axis to be $37^{\circ}$. However, the HI velocity field has a major axis position angle of $263^{\circ}\left(=83^{\circ}\right)$ overall, but with some irregularities. In the region that we measure the $\mathrm{H} \alpha$ velocities, the PA of the HI kinematics is of order $130^{\circ}$.

In one of our observations the slit was placed along the morphological major axis $39^{\circ}$. Other spectra were obtained along the morphological minor axis at $129^{\circ}$ and $\pm 45^{\circ}$ from the major axis at PAs of $84^{\circ}$ and $174^{\circ}$. The four slit positions are shown superposed on our $\mathrm{H} \alpha$ image in Figure 1. The center of the galaxy was taken as the center of middle-level isophotes in the V-band image.

\subsection{Ionized gas}

We show $\mathrm{V}_{\text {helio }}$ in the plane of the sky measured from [OIII] $\lambda 5007$ for NGC 1156 in Figure 6. Superposed on any rotational trends are large peculiar velocities in $\mathrm{V}_{\text {helio }}$. The peculiar velocities are due to high kinetic energy in the ionized gas that resulted from the energetic input of massive stars in HII regions. NGC 1156 is actively forming stars and contains many concentrations of massive stars. In our spectra we see deviations from rotation with line of sight velocities of order $12-20 \mathrm{~km} \mathrm{~s}^{-1}$ and extents along the slit of order $300 \mathrm{pc}$ to over 1000 pc. These are consistent with the expansion of giant and supergiant shells of ionized gas. Fairly systematic undulations seen in $\mathrm{PA} 39^{\circ}$ repeat in $\mathrm{PA} 174^{\circ}$ if the $\mathrm{PA} 174^{\circ}$ spectrum is flipped so that spatially nearby regions are superposed. Thus, these position angles reveal little of the overall rotation, and we have no alternative but to determine the major axis of rotation from the remaining spectra in PAs $84^{\circ}$ and $129^{\circ}$.

In Figure 6 we see evidence for ordered rotation of the ionized gas with a linear velocity gradient, but not at the PA one might expect to see it. For a rotating inclined axisymmetric 
disk, the steepest velocity gradient will be along the apparent major axis. The morphological major axis is $39^{\circ}$, but rotation at this PA is not obvious. We clearly see rotation in PAs $129^{\circ}$, the morphological minor axis, and PA $84^{\circ}$. The velocities in PA $174^{\circ}$ also appear relatively flat.

A weighted least squares fit to the points (excluding end points) from $\mathrm{r}=+29.9^{\prime \prime}$ to $\mathrm{r}=-36.6^{\prime \prime}(1.6 \mathrm{kpc})$ in $\mathrm{PA}=129^{\circ}$ is shown as the solid line in the panel of PA $129^{\circ}$ in Figure 6. Each of the spectra off the major axis should exhibit velocities which are the projected component of the major axis velocities. The fit to PA $129^{\circ}$ is shown transformed to the other PAs in the plane of the sky under the assumption that $129^{\circ}$ is the kinematical major axis. These are the solid lines in the other panels in Figure 6. A weighted least squares fit to the velocities in $\mathrm{PA}=84^{\circ}$ (excluding end points) from $\mathrm{r}=+42.2^{\prime \prime}$ to $\mathrm{r}=-19.2^{\prime \prime}$ is shown as the dashed line in panel $\mathrm{PA}=84^{\circ}$ in Figure 6 . This fit is also shown transformed to the other PAs under the assumption that $84^{\circ}$ is the line of nodes. These are the dashed lines in Figure 6.

We see that the velocity gradients in both PAs $84^{\circ}$ and $129^{\circ}$ are quite similar. Their similarity suggests that the line of nodes lies between $84^{\circ}$ and $129^{\circ}$, with the resulting velocity gradient being slightly steeper than the lines in Figure 6. Thus, the ionized gas kinematical major axis is not the same as the morphological major axis of the galaxy. If the kinematical axis is $129^{\circ}$, it is in fact the morphological minor axis of the galaxy but agrees with the HI kinematics in the inner part of the galaxy. However, if the ionized gas kinematical major axis is $84^{\circ}$, it is the same as the overall HI kinematic axis. Position angles are summarized in Table 4.

The velocity gradients that we measure at the two PAs are fairly similar. The fit to $\mathrm{PA}=129^{\circ}$ yields a gradient of $(0.58 \pm 0.08 / \sin i) \mathrm{km} \mathrm{s}^{-1} \operatorname{arcsec}^{-1}$. This becomes $(15.4 \pm$ $2.0 / \sin i)$ at our adopted distance. The fit to $\mathrm{PA}=84^{\circ}$ yields a gradient of $(0.51 \pm 0.04 / \sin i)$ $\mathrm{km} \mathrm{s}^{-1} \operatorname{arcsec}^{-1}$. This becomes $(13.5 \pm 1.1 / \sin i)$ at our adopted distance. If the true kinematical major axis lies mid-way between these two PAs, the velocity gradient would increase by a factor of 1.1 over the fit to PA $129^{\circ}$ and PA $84^{\circ}$ combined.

A comparison of the emission-line $V_{\text {helio }}$ with position-velocity slices through the HI data of Swaters (1999) is shown in Figure 7. There is a minimum in the HI in the center of the galaxy which is surrounded by higher column density HI complexes. Our ionized gas velocity measurements extend into the regions of higher gas column density. Given that there could be a small offset in $\mathrm{V}_{\text {sys }}$ between the optical and HI, the agreement between HI and ionized gas velocities in PAs $84^{\circ}$ and $174^{\circ}$ is strong; the ionized gas velocities generally fall along the peaks in the neutral gas. However, at PA $129^{\circ}$, the rotation of the ionized gas shows a steeper gradient than the HI. 
In PA $39^{\circ}$ the HI exhibits very broad velocity profiles at $r=+75^{\prime \prime}$. This region is the HI complex to the northeast of the galaxy center where the possible tidal arm of McIntyre (2002) ends. The optical velocities do not exhibit the low velocities seen in the HI at this position. However, our ionized gas velocities only just extend to this radius and so we may be missing the region of lower velocities seen in the HI.

\subsection{Stars}

The $\mathrm{V}_{\text {helio }}$ measured from the stellar absorption lines are plotted against position along the slit in Figure 8. In the figure we show the results from measuring along the spectra in $10.65^{\prime \prime}$ sums. This step size corresponds to intervals of $400 \mathrm{pc}$ in NGC 1156. The stellar velocities are compared to the HI and ionized gas velocities in Figure 7.

We see very weak rotation of the stars about the morphological major axis $\left(39^{\circ}\right)$, morphological minor axis $\left(129^{\circ}\right)$, and mid-way between $\left(84^{\circ}\right)$ to $1.6 \mathrm{kpc}$. Rotation about $39^{\circ}$ and $129^{\circ}$ seems marginally stronger than about $84^{\circ}$, but rotation about these two axes implies that neither can be the kinematical major axis since the other must be the minor axis and show no rotation if the motion is in an ordered disk. The only cicular-orbit model that makes physical sense is one in which the kinematical major axis is near PA $84^{\circ}$. In Figure 8 we show a least-squares fit (excluding end points) of $\mathrm{r}=43.3^{\prime \prime}(1.6 \mathrm{kpc})$ to $\mathrm{r}=-41.9^{\prime \prime}$ in PA $84^{\circ}$. We also show the consequences of assuming this PA is the major axis and transforming it to the other PAs in the plane of the sky. These are the solid lines in the figure. If $84^{\circ}$ is near the kinematical major axis, the minor axis is $174^{\circ}$, and the velocities in $\mathrm{PA} 174^{\circ}$ do appear to be flat.

The ionized gas rotation (the dashed line in Figure 6) is shown as the dashed line in Figure 8. The gradient of rotation in the NGC 1156 stars is $(0.18 \pm 0.06 / \sin i) \mathrm{km} \mathrm{s}^{-1}$ $\operatorname{arcsec}^{-1}$ or $(4.8 \pm 1.5 / \sin i) \mathrm{km} \mathrm{s}^{-1} \mathrm{kpc}^{-1}$. The slope is the same if we fit the points at PAs $39^{\circ}, 84^{\circ}$, and $129^{\circ}$ together. The velocity gradient in the stars is less than that in the ionized gas. However, the uncertainties in the stellar rotation velocities are large enough to allow both a velocity gradient that is comparable to that of the ionized gas and one in which there is no rotation of the stars.

In Figure 9 we plot the rotation velocities of the stars in the three PAs with rotation (that is, excluding PA $174^{\circ}$ ), projected onto the plane of the galaxy. We also include the rotation curve of the ionized gas and that determined from the HI velocity field (Swaters 1999) reflected about $\mathrm{r}=0$ for comparison. Note that the resolution of the HI is much lower $\left(\mathrm{HPBW}=30^{\prime \prime}\right)$ than that of the optical spectra. 


\section{Results: NGC 4449}

A deep V-band image of NGC 4449 has been analyzed by Hunter et al. (1999). They found that the PA of the major axis changes from the inner galaxy to the outer galaxy. In the inner galaxy $\left(<2.2^{\prime}\right)$ the major axis is at a PA of $46^{\circ}$, and in the outer galaxy $\left(>3.1^{\prime}\right)$ it is at $64^{\circ}$. This twisting of the isophotes is apparent in Figure 1 where we show the logarithm of the V-band image. A line is drawn along the major axis of the inner galaxy at $46^{\circ}$ to guide the eye. We obtained one spectrum with the slit placed along this axis. Other spectra were obtained along the minor axis of $136^{\circ}$ and $\pm 45^{\circ}$ from the major axis at $91^{\circ}$ and $1^{\circ}$. All four slit positions are shown superposed on the $\mathrm{H} \alpha$ image in Figure 1.

\subsection{Ionized Gas}

We plot $\mathrm{V}_{\text {helio }}$ measured from [OIII] $\lambda 5007$ in the four spectra of NGC 4449 in Figure 10. As for NGC 1156, there are regions where the deviations from rotation in $\mathrm{V}_{\text {helio }}$ are quite large. These peculiar velocities most likely are the result of the kinetic energy of the ionized gas that has resulted from the input from massive stars in the star-forming regions in the galaxy. NGC 4449 is well-known for the signatures of this energy input, including many ionized gas shells and filaments and broad $\mathrm{H} \alpha$ lines seen in high-dispersion spectra (Hunter 1982; Hunter \& Gallagher 1990, 1992, 1997; Hunter, Hawley, \& Gallagher 1993; Martin 1997). In our spectra we see deviations from rotation that are consistent with expansion velocities of order $20-100 \mathrm{~km} \mathrm{~s}^{-1}$ and extents along the slit up to $1200 \mathrm{pc}$. These are similar to those that we saw in NGC 1156. In addition we see emission along a single line of sight that is double-valued, with a large velocity difference $\left(\Delta \mathrm{V} \geq 100 \mathrm{~km} \mathrm{~s}^{-1}\right.$ in PA $1^{\circ}$ at $\mathrm{r} \sim-20^{\prime \prime}$, in $\mathrm{PA} 46^{\circ}$ at $\mathrm{r} \sim 40^{\prime \prime}$, and $\mathrm{PA} 136^{\circ}$ at $\mathrm{r} \sim-20^{\prime \prime}$ ). As in NGC 1156, the velocity extremes that we detect in nearby positions may arise from segments of the same structure. Hunter \& Gallagher (1997) point out that the center of the galaxy is the base of a $1.3 \mathrm{kpc}$ diameter supershell and coincident with X-ray emission. Most of the regions of peculiar velocities along the slit coincide with filaments or loops that are visible on the $\mathrm{H} \alpha$ image, some of which are identified by Hunter et al. (1993) and marked in Figure 10.

The input from massive stars in NGC 4449 is so energetic that it complicates the determination of the rotation. However, in $\mathrm{PA}=46^{\circ}$ we do see a velocity gradient that can be fit with a straight line. We fit the velocities, excluding the peculiar velocities, from $\mathrm{r}=110.1^{\prime \prime}(2.1 \mathrm{kpc})$ to $-62.7^{\prime \prime}$, and found a gradient of $(0.22 \pm 0.01 / \sin i) \mathrm{km} \mathrm{s}^{-1} \operatorname{arcsec}^{-1}$ (the solid line in Figure 10). For a distance of $3.9 \mathrm{Mpc}$, this gradient is $(11.8 \pm 0.7 / \mathrm{sin} i) \mathrm{km}$ $\mathrm{s}^{-1} \mathrm{kpc}^{-1}$. To see if the rotation curve in $\mathrm{PA}=46^{\circ}$ is consistent with circular rotation in a planar circular disk, we have transformed it to the other PAs in the plane of the sky (the 
solid lines in Figure 10). These projections fit the rotation data at the other PAs reasonably well, with considerable deviations due to the energetics of the ionized gas. Thus, the rotation of the ionized gas is consistent with a kinematic axis that is the same as the morphological major axis. Position angles are summarized in Table 4.

The $\mathrm{V}_{\text {helio }}$ of the ionized gas is shown superposed on position-velocity slices through the HI in Figure 11. The HI data are from Hunter et al. (1999), and we have extracted slices of the HI data cube at each of the optical PAs, summing over one HI beamwidth. We see that the ionized gas follows the rotation of the neutral gas. In some cases the peculiar velocities seen in the ionized gas also follow those in the HI; see, for example, PA $1^{\circ}$ at a radius of about $+1^{\prime}$. In some cases the ionized gas is not associated with peaks in the HI; see, for example, PA $91^{\circ}$ at an $\mathrm{r}$ of 0 . But, in many cases, the velocities of the ionized gas are close to or near HI peaks. Typical deviations of the ionized gas from the velocities of the HI peaks is of order $15-20 \mathrm{~km} \mathrm{~s}^{-1}$, with some as high as $50 \mathrm{~km} \mathrm{~s}^{-1}$. For the galaxy NGC 4214, which is very similar in luminosity to NGC 4449, Wilcots \& Thurow (2001) found velocity differences between the HI and $\mathrm{H} \alpha$ of order $50-100 \mathrm{~km} \mathrm{~s}^{-1}$. These large differences were associated with diffuse $\mathrm{H} \alpha$ in low column density HI gas, interpretted as champagne flows away from giant $\mathrm{H}$ II regions and into holes in the ISM.

\subsection{Stars}

The $\mathrm{V}_{\text {helio }}$ measured from the absorption lines are plotted against position along the slit in Figure 12. In the figure we show the results from measuring the absorption spectra summed in $10.65^{\prime \prime}$ steps along the slit. This corresponds to intervals of 200 pc in NGC 4449. The stellar rotation velocities are also plotted with the neutral and ionized gas in Figure 11. We have not detected rotation in NGC 4449 at any of the four position angles to a radius of $65^{\prime \prime}(1.2 \mathrm{kpc})$. The solid line in Figure 12 is the central velocity of $214 \mathrm{~km} \mathrm{~s}^{-1}$. The dashed line is the fit to the ionized gas rotation at PA $46^{\circ}$, and it is clear that we can rule out a stellar rotation comparable to that of the ionized gas. We set an upper limit on the rotation of the stars of $(3 / \sin i) \mathrm{km} \mathrm{s}^{-1} \mathrm{kpc}^{-1}$.

\section{Summary of observational results}

In NGC 2366 we have measured the rotation of the ionized gas to $114^{\prime \prime}(1.9 \mathrm{kpc})$. Rotation with a major axis PA of $30^{\circ}$, that of the optical galaxy, or of $45^{\circ}$, that of the HI kinematics, fit the observations equally well. The rotation gradient is linear (can be fit with 
a straight line) with a gradient of $(30 \pm 2 / \sin i) \mathrm{km} \mathrm{s}^{-1} \mathrm{kpc}^{-1}$. Peculiar velocities southwest of the galaxy center, where there are two supergiant HII regions, mask the rotation pattern there.

In NGC 1156 the ionized gas shows a rotation gradient that can be fit with a straight line to $42^{\prime \prime}(1.6 \mathrm{kpc})$. The major axis is $84^{\circ}-129^{\circ}$. The HI kinematics have a PA of order $130^{\circ}$ in the region the $\mathrm{H} \alpha$ is being measured, but a PA of order $83^{\circ}$ overall (Swaters 1999). However, the optical major axis has a PA of $39^{\circ}$, so the morphological and kinematic axes are misaligned. The gradient of the ionized gas is $(13 \pm 1 / \sin i) \mathrm{km} \mathrm{s}^{-1} \mathrm{kpc}^{-1}$. Rotation of the stars is weak, but linear to $43^{\prime \prime}(1.6 \mathrm{kpc})$. The most probable kinematic major axis of the stars is also $84^{\circ}$. The gradient is $(5 \pm 1 / \sin i) \mathrm{km} \mathrm{s}^{-1} \mathrm{kpc}^{-1}$.

In NGC 4449 we found that the ionized gas rotates in a manner consistent with a major axis of $46^{\circ}$, the same as the morphological major axis of the inner galaxy. The gradient is $(12 \pm 1 / \sin i) \mathrm{km} \mathrm{s}^{-1} \mathrm{kpc}^{-1}$ to $110^{\prime \prime}(2.1 \mathrm{kpc})$. The stars, on the other hand, show no ordered rotation, with an upper limit of $(3 / \sin i) \mathrm{km} \mathrm{s}^{-1} \mathrm{kpc}^{-1}$ to $65^{\prime \prime}(1.2 \mathrm{kpc})$.

In all three galaxies we have found that the ionized and neutral gas velocities are similar, but often with important differences. First, the rise of the rotation speeds with radius is steeper in the ionized gas than in the HI. This is easily understood as due to the large difference in resolution of the two data sets. The HI observations typically were made with a beam-size of order $30^{\prime \prime}$ while the optical spectra used a slit of $3^{\prime \prime}$. This beam-smearing effect can make the rotation curve deduced from the HI shallower (see, for example, Bosma 1981; Rubin et al. 1989; Blais-Ouellette et al. 1999; Swaters, Madore, \& Trewhella 2000).

Second, the major axes inferred from the kinematics of the different galactic components are not always the same, nor are they the same as the major axis of the optical morphology. The various PAs are collected in Table 4. In NGC 1156 the various gas and stellar kinematical axes are most likely the same, but this axis differs from the morphological major axis. In NGC 2366 the morphological and HI kinematical axes are different, and the ionized gas is equally consistent with either of these axes. In NGC 4449 the kinematical major axis of the ionized gas is coincident with the morphological major axis of the inner optical galaxy but differs from the HI kinematic axis.

In all three galaxies we can identify regions in which the ionized gas rotation velocity gradients can be approximated by a straight line. However, in many regions the true rotation is disguised by large superposed non-circular motions. The velocity gradient of the stars in NGC 1156 is also linear over the region of the galaxy that we measured rotation velocities. The gradients in the stars are lower than those in the ionized gas in both galaxies. 


\section{Discussion}

\subsection{NGC 1156}

\subsubsection{Misalignment of axes}

In NGC 1156 the stars and gas, both ionized and neutral, appear to rotate about the same axis. A line of nodes of about $84^{\circ}$ is consistent with all three components of the galaxy. However, this axis is different by about $45^{\circ}$ from the morphological major axis.

What does it mean when the line of nodes is misaligned with the morphological major axis? NGC 1156 has a rectangular appearance over much of the optical system. Although there is no twisting of isophotes or other tell-tale signs, this rectangular appearance could indicate the presence of a bar potential (Athanassoula et al. 1990). In modeling a disk galaxy, one usually assumes a plane circular thin disk which is inclined to the line of sight at a fixed angle. In a barred galaxy in which the bar extends most of the radius, the isophotes are not circular in the principle plane, the disk is not thin, and the orbits may precess, so the expectation in a well-behaved galaxy that the line of nodes and morphological axis are the same no longer applies. Identifying bar structures in irregular galaxies is complicated by the lack of symmetry provided by spiral arms coming out of the nucleus or bar (see discussion by Roye \& Hunter [2000] and Hunter et al. [2001]). Furthermore, the bar in NGC 1156 is a much larger fraction of the optical galaxy than is the case in spirals, even to the point where the bar is most of the optical galaxy. In spirals, on the other hand, the bar length is typically $\leq 0.3$ of the size of the stellar disk (Elmegreen \& Elmegreen 1985). However, bar structures are very stable and they can grow on timescales less than a Hubble time. So, while such large bars are not seen in more luminous galaxies, it is reasonable that a rectangular galaxy like NGC 1156 is dominated by a bar.

\subsubsection{Low stellar velocity gradient}

The apparent low velocity gradient in the stars compared to that of the ionized gas in NGC 1156 is also striking. The gradient in the stars is about 3 times lower than that of the ionized gas. However, the uncertainties in the stellar rotation velocities are large enough that we cannot rule out the possibility that the stellar gradient is nearly the same as that of the ionized gas. In Figure 8 the ionized gas velocity gradient is superposed on the stellar rotation velocities. In all but PA $84^{\circ}$ the ionized gas velocity gradient fits within the error

bars of the stellar points. By the same argument, of course, we cannot rule out the possibility that the stellar velocity gradient is lower, even zero. 
If the velocity gradient of the stars is lower than that of the gas, it is possible that more of the kinetic energy of the stars is in random motions rather than ordered rotation compared to the gas. The rotation velocity of the ionized gas is $(15.9 \pm 1.3 / \sin i) \mathrm{km} \mathrm{s}^{-1}$ at a radius of $31.3^{\prime \prime}$ where we measure a rotation velocity for the stars of $(5.7 \pm 1.8 / \sin i) \mathrm{km} \mathrm{s}^{-1}$. If the galaxy is a disk with the ionized gas defining the circular rotation speed, we can estimate the azimuthal velocity dispersion of the stars from $\sigma_{\phi}^{2} \sim v_{\text {gas }}^{2}-v_{\text {stars }}^{2}$ (see discussion in $\S 4.2 .1$ (a) of Binney \& Tremaine 1987). This would yield a stellar velocity dispersion of order $(14.8 / \sin i)$ $\mathrm{km} \mathrm{s}^{-1}$ to compensate for the lower observed rotation velocity, assuming the stars and gas are in the same disk. For an inclination of $51^{\circ}$, determined for the HI velocity field (Swaters 1999), $\sigma_{\phi}$ would be about $19 \mathrm{~km} \mathrm{~s}^{-1}$, comparable to the value predicted for the $\mathrm{M}_{B}$ of the galaxy (Bottema 1993, Swaters 1999, van der Marel et al. 2002).

However, there is an inconsistency in this argument. Although the absorption features that we targeted are due to $\mathrm{F}$-K-type stars, these may be dominated by stars less than about 1 Gyr of age. The high surface brightness region that we observed is blue and the V-band is dominated by stars $\leq 1$ Gyr old. It would be hard to impart a large level of randomization to the velocities of the stars on this timescale. Also, there is no obvious structure at the center of the galaxy to scatter the stars.

\subsection{NGC 4449}

\subsubsection{No measureable rotation of the stars}

NGC 4449 was known to be peculiar. Large streamers of HI that encircle the galaxy indicate that another galaxy has interacted with NGC 4449 sometime in the past (Hunter et al. 1998). Furthermore, the HI gas in the inner $2^{\prime}$ of the galaxy appears to be counterrotating with respect to the gas at larger radii, a clear signature of an interaction. The stars do not correspond to either gas system: we detected no ordered rotation to $<3 / \sin i \mathrm{~km} \mathrm{~s}^{-1}$ $\mathrm{kpc}^{-1}$ to $1.2 \mathrm{kpc}$. As for NGC 1156, the uncertainties in the stellar rotation velocities are relatively high, but the maximum allowed velocity gradient is still 4 times lower than the gradient observed in the ionized gas. As Figure 12 shows, it is unlikely that the stars are rotating at the same rate as the gas.

What does it mean then when the stars do not rotate but the gas does? One possibility is that the stars, in contrast to the gas, lie in a disk that is nearly face-on. An inclination of $45^{\circ}$ is inferred from $b / a$ in the outer isophotes of the galaxy under the assumption that the intrinsic axis ratio is 0.3. However, NGC 4449, like NGC 1156, is rectangular in shape, and there is a twisting of the $\mathrm{V}$-band isophotes from the central rectangle to the outer galaxy. 
Hunter et al. (1999) interpret these characteristics as a bar structure that has a length that is $90 \%$ of $\mathrm{D}_{25}$. If the galaxy is dominated by a bar, the observed $b / a$ is not a valid indicator of the inclination of the galaxy.

\subsubsection{A model for the gas kinematics}

If the stars are in a disk seen nearly face-on, how would we understand the kinematics of the gas? One explanation is that the HI disk is highly warped (Swaters 1999). We explored this possibility by constructing a model for the gas in which the gas is in orbits of constant inclination, but with precession-induced twisting of the line of nodes.

We used fits to the HI velocity field of NGC 4449 to guide the models. Hunter et al.(1998, 1999) have presented such fits, but, because of the complex HI velocity field in this galaxy, we have now calculated additional fits to remind ourselves of the degree of uncertainty. We fit the data from $15^{\prime \prime}$ to $2^{\prime}$ in summed annular steps of $15^{\prime \prime}$ and from $135^{\prime \prime}$ to $12^{\prime}$ in steps of $45^{\prime \prime}$. Although we explored a variety of parameters, in the final fit we fixed the center as the optical center of the galaxy. First, we allowed the inclination, PA, and rotation speed to vary with each annulus. Then we fixed the inclination and allowed only the PA and rotation speed to vary. We found an inclination of $71 \pm 9^{\circ}$ and a PA that varied between $80^{\circ}$ and $105^{\circ}$. These values are close to those of Hunter et al. (1999) for the inner galaxy - an inclination of $77^{\circ}$ at a position angle of $72^{\circ}$. Thus, the present model uses a line of nodes of $80^{\circ}$ in the central $2^{\prime}$. For the outer galaxy, we found an inclination of $66 \pm 17^{\circ}$ with the position angle settling down to $253 \pm 4^{\circ}$ beyond $6^{\prime}$. Hunter et al. (1998) had found an inclination of $60^{\circ}$ and a position angle of $230 \pm 17^{\circ}$. The models of Theis \& Kohle (2001) that successfully reproduce the extended gas streamers constrain the outer gas disk to an inclination of $50-70^{\circ}$. Our model uses a line of nodes of $-110^{\circ}\left(=250^{\circ}\right)$ in the outer regions $\left(\mathrm{r}>5^{\prime}\right)$.

The model that we present here extends to a radius of $10^{\prime}$, has a disk inclination of $68^{\circ}$, and uses a systemic velocity of $214 \mathrm{~km} \mathrm{~s}^{-1}$. The maximum circular velocity is $80 \mathrm{~km} \mathrm{~s}^{-1}$, and the velocity ramps linearly from 0 to $80 \mathrm{~km} \mathrm{~s}^{-1}$ between $\mathrm{r}=0$ and $\mathrm{r}=2.5^{\prime}$. The line of nodes in the inner $2^{\prime}$ is $80^{\circ}$ and decreases linearly with radius to $-110^{\circ}$ at a radius of $5^{\prime}$. A three dimensional representation of the model orbits is shown in Figure 13. Physically, this model corresponds to a disk of gas in tilted orbits which precess around the minor axis of an oblate gravitational potential. Precession periods are shorter at smaller radii, so the line of nodes regresses, and the gas disk twists. The line of nodes precesses retrograde to rotation in an oblate potential, and since precession periods are shorter at smaller radii, the line of nodes develops a leading twist with radius. Such orbits can be stable until cloud-cloud collisions produce dissipation, so they can last a long time. 
We compare the model to the observations in Figure 14. Here we reproduce the HI position-velocity plots from Figure 11 and superpose simulated observations of the model at $15^{\prime \prime}$ intervals at our four PAs. One can see that the model reproduces well the observed HI rotation velocities including the counter-rotation in the inner galaxy. It predicts broad or multiple profiles in the $\mathrm{HI}$ in places: particularly at $\pm 2^{\prime}-2.5^{\prime}$ along $\mathrm{PA} 46^{\circ}$ and $\pm 1.5^{\prime}-2^{\prime}$ along PA $91^{\circ}$. Plots of HI profiles in the inner galaxy do show regions with broad and doublepeaked profiles (broad profiles are easily seen in the position-velocity plots of Figure 14), although not exclusively at the regions predicted by the models, which do not take account of beam-smearing in the HI observations. We conclude that this simple model is a plausible representation of the orbits of the gas in NGC 4449.

Such tilted orbits would result from tipping gas in at an angle to the stellar disk. Most probably the gas was acquired from a passing or merging object. Since there are other signs that NGC 4449 was involved in a past interaction, we can understand the gas in these peculiar orbits as resulting from that interaction or a second event. The models of Theis \& Kohle (2001) that reproduce the extended streamers as resulting from an interaction with DDO 125 involve no mass transfer and do not reproduce the apparent counter-rotating gas system in the center. If the acquired gas was simply gas in NGC 4449 pulled out by a passing galaxy and now settling back in, we would expect stars to have been pulled out into tilted orbits also, which we do not see. Thus, an accretion event is required.

Hunter et al. (1999) had argued that NGC 4449 before the interaction had to have been a late-type galaxy and, therefore, likely gas-rich. This argument was based on the ensemble of characteristics including small $\mathrm{M}_{B}$, small optical scale length, lack of a bulge, small rotation speed, current high gas content, and low metallicity. If NGC 4449 was relatively gas-rich to begin with, the acquired gas is falling onto other gas that was already there, presumably in the same orbits as the stars. The original disk gas could have lost angular momentum in the encounter and been pushed further in, and is now a small inner nearly-face-on disk. However, the HI observations do not allow such a face-on disk of original material to be very massive; the entire HI mass within $\mathrm{r}<2^{\prime}$ is only $3 \times 10^{8} \mathrm{M}_{\odot}$ (Hunter et al. 1999), and the center of the galaxy represents a minimum in the HI distribution.

In the models, the twisting of the line of nodes of the gas takes place from a radius of $2^{\prime}$ to a radius of $5^{\prime}$. The region $2^{\prime}<\mathrm{r}<3^{\prime}$ is a special place in NGC 4449 in several other ways as well. First, the morphological major axis changes PA in this region. The PA of the morphological major axis rotates from $46^{\circ}$ for the rectangular inner galaxy $\left(\mathrm{r}<2.2^{\prime}\right)$ to $64^{\circ}$ at $r>3.1^{\prime}$. If the rectangular structure of the galaxy is a bar, this region represents the end of the bar potential. Second, there are large HI complexes located at a radius of $1.5^{\prime}-2^{\prime}$. These HI complexes are part of a large HI ring that surrounds the optical galaxy. Gas could be 
collecting there both because it is trapped near the end of the bar, and because infalling gas is piling on with an increased number of cloud-cloud collisions. The cloud-cloud collisions would further account for the star formation that is currently seen there.

Sung et al. (1998) have argued that irregular galaxies are triaxial in shape. In NGC 4449, the twisting of the isophotes (de Zeeuw 1984) and the rectangular shape (Binney \& Tremaine 1987) could be consistent with a triaxial shape with stars on box and loop orbits. However, few elliptical galaxies, which as a class include triaxial systems, are as boxy as NGC 4449 (See Peleier et al. 199; Figure 2 of Ryden et al. 1999; Figure 6.1 of Sparke \& Gallagher 2000; but also see Figure 2 of Jarvis 1987), although bar structures can be (Athanassoula et al. 1990). Since we can fit the observations of NGC 4449 with a reasonable model involving gas in a warped plane relative to the stars, and have an obvious mechanism for putting gas into these orbits, we do not embrace more complicated explanations.

\subsection{Summary of interpretation}

We measure a weak velocity gradient in the stellar disk of NGC 1156 and no rotation of the stars in NGC 4449. We find that we can understand the stellar and gas kinematics of NGC 1156 and NGC 4449 as disk systems dominated by bar potentials. In both galaxies the bar contains a large fraction of the light of the optical galaxy.

The low stellar velocity gradient of the stars in NGC 1156 is consistent with the velocity gradient of the gas within the observational uncertainties. A consistent and satisfying picture of NGC 1156 results if the stellar velocity gradient is close to that of the ionized gas and all components are in circular rotation about a common axis. The misalignment of the kinematical axis with the morphological axis is the result of a large bar dominating the disk luminosity.

In NGC 4449, where the stars rotate much more slowly than the gas, we can qualitatively explain the observations by assuming that the stars are in a disk seen nearly face-on, while the gas lies in a tilted disk with precession-induced twisting of the line of nodes. Most likely, the gas in this tilted disk was acquired from an interaction or possibly a merger, consistent with other signs of a past interaction in the galaxy.

Further clarification and refinement of our picture of the stellar kinematics in both NGC 1156 and NGC 4449 will have to await a measurement of the stellar velocity dispersions in these systems.

We wish to thank Phil Massey, Skip Andree, and other members of the KPNO staff for 
help in making the $4 \mathrm{~m}$ telescope and RC spectrograph perform so well for us. Support for this work was provided to DAH by the Lowell Research Fund and in part by the National Science Foundation through grant AST-9616940 to DAH and grant AST-0098419 to LSS. 
Table 1. Characteristics of the galaxies.

\begin{tabular}{|c|c|c|c|c|c|c|}
\hline $\begin{array}{l}\text { Galaxy } \\
\text { (1) }\end{array}$ & $\begin{array}{c}\mathrm{D} \\
(\mathrm{Mpc}) \\
(2)\end{array}$ & $\begin{array}{c}\mathrm{E}(\mathrm{B}-\mathrm{V})_{f} \\
(3)\end{array}$ & $\begin{array}{c}\mathrm{M}_{B, 0} \\
(4)\end{array}$ & $\begin{array}{c}\mathrm{D}_{25} \\
(\mathrm{kpc}) \\
(5)\end{array}$ & $\begin{array}{c}\text { RA } \\
(2000) \\
(6)\end{array}$ & $\begin{array}{c}\text { DEC } \\
(2000) \\
(7)\end{array}$ \\
\hline NGC 1156 & 7.8 & 0.165 & -18.0 & 7.5 & 25942.3 & 251415 \\
\hline NGC 2366 & 3.4 & 0.043 & -16.5 & 5.2 & 72852.3 & 691230 \\
\hline NGC 4449 & 3.9 & 0.000 & -18.2 & 7.2 & 122811.0 & 440536 \\
\hline
\end{tabular}

Note. - Column notes: (2) For NGC 1156 and NGC 4449 distances are determined from radial velocities corrected to the Galactic Standard of Rest (RC3) and a Hubble constant of $65 \mathrm{~km} \mathrm{~s}^{-1} \mathrm{Mpc}^{-1}$. For NGC 2366 the distance comes from cepheid light curves (Tolstoy et al. 1995). (3) Foreground reddening from Burstein \& Heiles (1984). We use the extinction curve of Cardelli, Clayton, \& Mathis (1989) to correct for extinction. (4) Integrated $\mathrm{M}_{B}$ corrected for extinction. Data for NGC 1156 from RC3; data for NGC 2366 from Hunter et al. (2001); data for NGC 4449 from Hunter et al. (1999). (5) The diameter of the galaxy measured to an extinctioncorrected B-band surface brightness of 25 magnitudes $\operatorname{arcsec}^{-2}$ (NGC 1156: RC3; NGC 2366: Hunter et al. (2001); NGC 4449: Hunter et al. (1999). (6-7) The center of the galaxy determined from V-band images and used to place the center of the slit. Units of RA are hours, minutes, seconds; units of DEC are degrees, arcminutes, and arcseconds. 
Table 2. Template stars observed on each night.

\begin{tabular}{|c|c|c|c|}
\hline Night & Star & Type & $\mathrm{V}_{\text {helio }}{ }^{\mathrm{a}}\left(\mathrm{km} \mathrm{s}^{-1}\right)$ \\
\hline \multirow[t]{4}{*}{1} & HD 10380 & K3III & $\cdots$ \\
\hline & HD 41636 & G9III & $\cdots$ \\
\hline & HD 132142 & $\mathrm{~K} 1 \mathrm{~V}$ & $\cdots$ \\
\hline & HD 145328 & K0III-IV & $\ldots$ \\
\hline \multirow[t]{2}{*}{2} & HD 9138 & K4III & +35.4 \\
\hline & HD 5211 & F4III & $\ldots$ \\
\hline \multirow[t]{2}{*}{3} & HD 4338 & K3III & -28.3 \\
\hline & HD 107328 & K0.5IIIb & +35.7 \\
\hline \multirow[t]{3}{*}{4} & HD 1069 & K2I & $\ldots$ \\
\hline & HD 1400 & K5I & $\cdots$ \\
\hline & HD 18884 & M1.5III & -25.8 \\
\hline
\end{tabular}

${ }^{a}$ Radial velocity standards are taken from the United States Nautical Almanac (2000). 
Table 3. Central velocities $\mathrm{V}_{\text {sys }}\left(\mathrm{km} \mathrm{s}^{-1}\right)$.

\begin{tabular}{|c|c|c|c|c|c|c|c|}
\hline Galaxy & Slit PA & Ionized Gas & Stars & $\begin{array}{l}\Delta \mathrm{V}_{\text {sys }} \\
\text { Ionized Gas }\end{array}$ & $\begin{array}{l}\Delta \mathrm{V}_{\text {sys }} \\
\text { Stars }\end{array}$ & Average & $\mathrm{HI}^{\mathrm{a}}$ \\
\hline \multirow[t]{4}{*}{ NGC 1156} & 39 & $383.4 \pm 2.1$ & $379.7 \pm 0.4$ & -2.2 & +1.5 & $381.2 \pm 2.1$ & 371 \\
\hline & 84 & $\ldots$ & $\ldots$ & +0.0 & +7.9 & & \\
\hline & 129 & $382.7 \pm 1.4$ & $371.5 \pm 1.8$ & -1.5 & -0.3 & & \\
\hline & 174 & $\ldots$ & $378.5 \pm 0.7$ & -3.3 & +2.7 & & \\
\hline \multirow[t]{4}{*}{ NGC 2366} & 30 & $95.2 \pm 2.3$ & $\ldots$ & +0.8 & $\ldots$ & $96.0 \pm 0.7$ & $98-104$ \\
\hline & 27 & $96.4 \pm 2.3$ & $\ldots$ & -0.4 & $\ldots$ & & \\
\hline & 63 & $\ldots$ & $\ldots$ & -0.5 & $\ldots$ & & \\
\hline & 120 & $96.5 \pm 0.9$ & $\ldots$ & -1.8 & $\ldots$ & & \\
\hline \multirow[t]{4}{*}{ NGC 4449} & 1 & $\ldots$ & $212.2 \pm 1.4$ & -4.8 & +1.8 & $214.0 \pm 2.5$ & $208-214$ \\
\hline & 46 & $218.2 \pm 1.0$ & $212.4 \pm 1.0$ & -4.1 & +1.7 & & \\
\hline & 91 & $\ldots$ & $213.1 \pm 1.5$ & -2.8 & +1.0 & & \\
\hline & 139 & $\ldots$ & $214.4 \pm 1.7$ & -8.5 & -0.3 & & \\
\hline
\end{tabular}

${ }^{a}$ References for HI: NGC 1156 - Swaters 1999; NGC 2366-Hunter et al. 2001 and references therein; NGC 4449-Hunter et al. 1998, 1999. 
Table 4. Position angles.

\begin{tabular}{lrc}
\hline \hline \multirow{2}{*}{ Galaxy } & \multicolumn{1}{c}{ Data } & PA \\
& \multicolumn{1}{c}{ (deg) } \\
\hline \multirow{2}{*}{ NGC 1156 } & V-band & 39 \\
& HI kinematics (overall) & 83 \\
& HI kinematics (inner) & 130 \\
& Ionized gas kinematics & $84-129$ \\
NGC 2366 & Stellar kinematics & 84 \\
& V-band & 32 \\
& Ionized gas kinematics & 46 \\
NGC 4449 & V-band $\left(\mathrm{r}<2.2^{\prime}\right)$ & 46 \\
& V-band $\left(\mathrm{r}>3.1^{\prime}\right)$ & 64 \\
& HI kinematics $\left(\mathrm{r}<2^{\prime}\right)$ & $80-105$ \\
& HI kinematics $\left(6^{\prime}<\mathrm{r}<12^{\prime}\right)$ & 73 \\
& Ionized gas kinematics & 46 \\
\hline
\end{tabular}




\section{REFERENCES}

Aparicio, A., Cepa, J., Gallart, C., Castañeda, H. O., Chiosi, C., Bertelli, G., Mas-Hesse, J. M., Muñoz-Tuñón, C., Telles, E., Tenorio-Tagle, G., Diaz, A. I., García-Vargas, L. M., Garzón, F., González-Delgado, R. M., Pérez, E., Rodríguez-Espinosa, J. M., Terlevich, E., \& Televich, R. J. 1995, AJ, 110, 212

Athanassoula, E., Morin, S., Wozniak, H., Puy, D., Pierce, M. J., Lombard, J., \& Bosma, A. 1990, MNRAS, 245, 130

Bajaja, E., Huchtmeier, W. K., \& Klein, U. 1994, A\&A, 285, 388

Binggeli, B., \& Popescu, C. C. 1995, A\&A, 298, 63

Binney, J., \& Tremaine, S. 1987, Galactic Dynamics (Princeton:Princeton University Press)

Blais-Ouellette, S., Carignan, C., Amram, P., \& Côté, S. 1999, AJ, 118, 2123

Bosma, A. 1981, AJ, 86, 1825

Bottema, R. 1993, A\&A, 27516

Braun, R. 1995, A\&AS, 114, 409

Burstein, D., \& Heiles, C. 1984, ApJS, 54, 33

Cardelli, J. A., Clayton, G. C., \& Mathis, J. S. 1989, ApJ, 345, 245

de Vaucouleurs, G. 1975, in Galaxies and the Universe, edited by A. Sandage, M. Sandage, and J. Kristian (Univ Chicago Press: Chicago), p 557

de Vaucouleurs, G., de Vaucouleurs, A., Corwin, H., Buta, R., Paturel, G., \& Fouqué, P. 1991, Third Reference Catalogue of Bright Galaxies (New York:Springer-Verlag) ( $\mathrm{RC} 3)$

de Zeeuw, T. 1984, in Formation and Evolution of Galaxies and Large Structures in the Universe, (Dordrecht:Reidel), p 293

Drissen, L., Roy, J.-R., Robert, C., Devost, D., \& Doyon, R. 2000, AJ, 119, 688

Elmegreen, B. G., \& Elmegreen, D. M. 1985, ApJ, 288, 438

Hodge, P. W., \& Hitchcock, J. L. 1966, PASP, 78, 79

Huchtmeier, W. K., Seiradakis, J. H., \& Materne, J. H. 1981, A\&A, 102, 134

Hunter, D. A. 1982, ApJ, 260, 81

Hunter, D. A. 1997, PASP, 109, 937

Hunter, D. A., Elmegreen, B. G., \& van Weorden, H. 2001, ApJ, 556, 773

Hunter, D. A., \& Gallagher, J. S. 1990, ApJ, 362, 480 
Hunter, D. A., \& Gallagher, J. S. 1992, ApJ, 391, L9

Hunter, D. A., \& Gallagher, J. S. 1997, ApJ, 475, 65

Hunter, D. A., Hawley, W. N., \& Gallagher, J. S. 1993, AJ, 106, 1797

Hunter, D. A., van Woerden, H., \& Gallagher, J. S. 1999, AJ, 118, 2184

Hunter, D. A., Wilcots, E. M., van Woerden, J. S., \& Kohle, S. 1998, ApJ, 495, L47

Jacoby, G. H., Hunter, D. A., \& Christian, C. A. 1984, ApJS, 56, 257

Jarvis, B. 1987, AJ, 94, 30

Karachentsev, K., Musella, I., \& Grimaldi, A. 1996, A\&A, 310, 722

Kaufman \& Sugar 1986, J Chem Phy Ref Data, Vol. 15, No. 1

Martin, C. L. 1997, ApJ, 491, 561

McIntyre, V. 2002, private communication

Odewahn, S. C. 1989, PhD thesis, University of Texas

Patterson, R. J., \& Thuan, T. X. 1996, ApJS, 107, 103

Peletier, R. F., Davies, R. L., Illingworth, G. D., Davis, L. E., \& Cawson, M. 1990, AJ, 100, 1091

Roy, J.-R., Aubé, M., McCall, M. L., \& Dufour, R. J. 1992, ApJ, 386, 498

Roye, E. W., \& Hunter, D. A. 2000, AJ, 119, 1145

Rubin, V. C., Hunter, D. A., \& Ford, W. K. 1991, ApJS 76153

Rubin, V. C., Kenney, J. D., Boss, A. P., \& Ford, W. K., Jr. 1989, AJ, 98, 1246

Ryden, B. S., Terndrup, D. M., Pogge, R. W., \& Laurer, T. R. 1999, ApJ, 517, 650

Simkin, S. 1974, A\&A, 31, 129

Skillman, E. D., Terlevich, R., Teuben, P. J., \& van Woerden, H. 1988, A\&A, 198, 33

Sparke, L. S., \& Gallagher, J. S. 2000, Galaxies in the Unvierse. An Introduction (Cambridge:Cambridge Univ Press)

Statler, T. 1995, AJ, 109, 1371

Statler, T. S., \& Fry, A. M. 1994, ApJ, 425, 481

Statler, T. S., \& Smecker-Hane, T. 1999, AJ, 117, 839

Statler, T. S., Smecker-Hane, T., \& Cecil, G. N. 1996, AJ, 111, 1512

Staveley-Smith, L., Davies, R. D., \& Kinman, T. D. 1992, MNRAS, 258, 334 
Sung, E.-C., Han, C., Ryden, B. S., Patterson, R. J., Chun, M.-S., Kim, H.-I., Lee, W.-B., \& Kim, D.-J. 1998, ApJ, 505, 199

Swaters, R. 1999, PhD thesis, Rijksuniversiteit Groningen

Swaters, R. A., Madore, B. F., \& Trewhella, M. 2000, ApJ, 531, L107

Theis, Ch., \& Kohle, S. 2001, A\&A, 370, 365

Tolstoy, E., Saha, A., Hoessel, J. G., \& McQuade, K. 1995, AJ, 110, 1640

Tomita, A., Ohta, K., Nakanishi, K., Takeuchi, T. T., \& Saitō, M. 1998, AJ, 116, 131

Tonry, J., \& Davis, M. 1979, AJ, 84, 1511

United States Nautical Almanac Office, 2000, Astronomical Almanac (Washington, DC:US Government Printing Office)

van den Bergh, S. 1988, PASP, 100, 344

van der Marel, R. P., Alves, D. R., Hardy, E., \& Suntzeff, N. B. 2002, AJ, submitted van Woerden, H., Bosma, A., \& Mebold, U. 1975, in La Dynamique des Galaxies Spirales, ed. L. Weliachew (Paris:Editions du Centre National de la Recherche Scientifique), p 483

van Zee, L. 2000, AJ, 119, 2757

Wevers, B. M. H. R., van der Kruit, P. C., \& Allen, R. J. 1986, A\&AS, 66, 505

Wilcots, E. M., \& Thurow, J. C. 2001, ApJ, 555, 758 
Fig. 1. - False-color representation of the logarithm of the V-band and $\mathrm{H} \alpha$ images of our galaxies. We show the logarithm of the $\mathrm{V}$-band image in order to allow comparison of different surface brightness levels. In the V-band images a line is drawn along the morphological major axis. All four PAs observed in each galaxy are shown in the $\mathrm{H} \alpha$ images. The circle marks the center of the galaxy as given in Table 1. The ticks along the lines that show the PAs mark the extent over which we measured the absorption features (in the V-band images) and emission features (in the $\mathrm{H} \alpha$ images). The V-band and $\mathrm{H} \alpha$ images for NGC 2366 come from Hunter et al. (2001) and for NGC 4449 from Hunter et al. (1999). The V-band image of NGC 1156 was kindly obtained for us by P. Massey with a Tektronic $2048 \times 2048$ CCD on the KPNO $4 \mathrm{~m}$ telescope. The $\mathrm{H} \alpha$ image of NGC 1156 was obtained with the Perkins $1.8 \mathrm{~m}$ telescope at Lowell Observatory in 1995, using a TI CCD and a $32 \AA$ FWHM interference filter and $95 \AA$ off-band filter.

Fig. 2.- Line-of-sight heliocentric radial velocities $V_{\text {helio }}$ measured from the emission line [OIII] $\lambda 5007$ in the spectra of NGC 2366. Position along the slit is measured from the center of the galaxy, and positive numbers refer to the side of the slit that is in the direction of the given PA. Model 1 (major axis of $30^{\circ}$, solid line), and Model 2 (major axis of $45^{\circ}$, dashed line) are discussed in $\$ 4$. The lines are found from linear fits over the radial extent of the lines shown. The giant HII regions NGC 2363 and NGC 2366-III (Drissen et al. 2000) are marked along with a region of filaments near NGC 2363 (Hunter et al. 1993).

Fig. 3.- Line-of-sight heliocentric radial velocities of the ionized gas are plotted on contours of the neutral HI gas in NGC 2366. The HI position-velocity plots are taken from data presented by Hunter et al. (2001). The HI was summed over $35^{\prime \prime}$, approximately one beamwidth, and contours are $0.88,3.5,11.8$, and $35.3 \times 10^{20} \mathrm{~cm}^{-2}$. There could be a small offset in $\mathrm{V}_{\text {sys }}$ between the optical and $\mathrm{HI}$ (see Table 3 ).

Fig. 4.- Emission-line [OIII] $\lambda 5007$ line-of-sight heliocentric velocities in NGC 2366 in PA $27^{\circ}, 30^{\circ}$, and $63^{\circ}$ are superposed over the limited region of $+125^{\prime \prime}<\mathrm{r}<-50^{\prime \prime}$. The solid line is the fit to the data and is designated Model 2 (kinematical major axis of $45^{\circ}$ ).

Fig. 5.- Rotation velocity $\mathrm{V}_{\text {rot }}$ plotted against distance from the center of the galaxy in the plane of the galaxy for NGC 2366. The points are the data at PA $27^{\circ}$ and $30^{\circ}$ converted to rotation velocity using a position angle of $30^{\circ}$ (Model 1 ) and an inclination of $72^{\circ}$. The solid line is the Model 1 fit to the data $\left(\right.$ major axis $\mathrm{PA}=30^{\circ}$ ), and the tilted dashed line is the Model 2 fit to the data (major axis $\mathrm{PA}=45^{\circ}$ ). The lengths of the lines indicate the regions over which the fits were made. The long-dashed, curved line is the HI rotation curve (Hunter et al. 2001). The short-dashed horizontal line at a rotation speed of 0 is intended to assist the eye. 
Fig. 6. - Line-of-sight heliocentric radial velocities $\mathrm{V}_{\text {helio }}$ measured from the emission lines of the ionized gas in each spectrum of NGC 1156. The position angle of each spectrum is given; the top left panel is the morphological major axis. Position along the slit is measured from the center of the galaxy, seen as the intersection of all the slits in Figure 1, and positive numbers refer to the direction of the given PA. The solid line is a fit to the velocities in $\mathrm{PA}=129^{\circ}$ from $\mathrm{r}=+29.9^{\prime \prime}$ to $\mathrm{r}=-36.6^{\prime \prime}$. The length of the line indicates the region over which the fit was made. The solid lines in the other PAs are the fit at PA=129 transformed to the given PA in the plane of the sky assuming a kinematical major axis of $129^{\circ}$ and an inclination angle of $35^{\circ}$. The dashed line is the fit to the data in PA $84^{\circ}$ from $\mathrm{r}=+42.2^{\prime \prime}$ to $\mathrm{r}=-19.2^{\prime \prime}$ and the transformation of that fit to the other PAs in the plane of the sky assuming the kinematic major axis is along PA $84^{\circ}$. The place where the PA $39^{\circ}$ slit cuts through the large HII complex to the southwest is marked.

Fig. 7.- Line-of-sight heliocentric radial velocities $\mathrm{V}_{\text {helio }}$ of the ionized gas and stars are compared to that of the neutral HI gas (contours) in NGC 1156. The HI position-velocity plot is taken from data presented by Swaters (1999). The HI was summed over $30^{\prime \prime}$, approximately one beam-width, and contours are 1.0, 2.1, 3.2, and $4.3 \times 10^{20} \mathrm{~cm}^{-2}$. There could be a small offset in $\mathrm{V}_{\text {sys }}$ between the optical and $\mathrm{HI}$ (see Table 3 ).

Fig. 8.- Line-of-sight heliocentric radial velocities $\mathrm{V}_{\text {helio }}$ measured from the stellar absorption lines in each spectrum of NGC 1156. The absorption measurements are $10.65^{\prime \prime}$ sums stepped along the slit. The position angle of each spectrum is given; the top left panel is the morphological major axis. Position along the slit is measured from the center of the galaxy, seen as the intersection of all the slits in Figure 1, and positive numbers refer to the direction of the given PA. The solid lines are the rotation curve determined from a least-squares fit to the points in $\mathrm{PA}=84^{\circ}$ from $\mathrm{r}=+43.3^{\prime \prime}(1.6 \mathrm{kpc})$ to $\mathrm{r}=-41.9^{\prime \prime}$ (the length of the line) and transformed to the other position angles in the plane of the sky, assuming an inclination of $35^{\circ}$. The dashed line is the fit to the rotation of the ionized gas (the dashed line from Figure $6)$, plotted here for comparison.

Fig. 9.- Rotation velocity $\mathrm{V}_{\text {rot }}$ plotted against distance from the center of the galaxy in the plane of the galaxy for the stars in NGC 1156. The solid horizontal line at a rotation speed of 0 is intended to assist the eye. The solid line is the fit to PA $84^{\circ}$ transformed to the plane of the galaxy using an inclination of $35^{\circ}$. The length of the line indicates the region over which the fit was performed. The dashed line is the rotation curve determined from the HI velocity field (Swaters 1999) reflected about $\mathrm{r}=0$; from the fit to the HI velocity field the PA is $83^{\circ}$ and the inclination is $51^{\circ}$. The error bar in the lower right corner gives the median uncertainty in the velocities. 
Fig. 10.- Line-of-sight heliocentric radial velocities $\mathrm{V}_{\text {helio }}$ measured from the emission lines of the ionized gas in the spectra of NGC 4449. The position angle of each spectrum is given; the top left panel is the morphological major axis for $\mathrm{r}<2.2^{\prime}$ determined from the V-band image. Position along the slit is measured from the center of the galaxy, seen as the intersection of all the slits in Figure 1, and positive numbers refer to the direction of the given PA. The solid line in $\mathrm{PA}=46^{\circ}$ is a least-squares fit to the portion of the data with a linear rotation gradient (excluding end points) from $r=+110.1^{\prime \prime}$ to $r=-62.7^{\prime \prime}$. The line is shown only as long as the region fit. The solid lines in the other PAs are transformations of this fit to the given PA in the plane of the sky under the assumption of an inclination angle of $45^{\circ}$. Peculiar velocities that are concident with obvious shells and filaments are marked; features identified as "HHG" are catalogued in Hunter, Hawley, \& Gallagher (1993).

Fig. 11. - Line-of-sight heliocentric radial velocities of the ionized gas and stars are compared to that of the HI in NGC 4449. The HI position-velocity plots are taken from data presented by Hunter et al. (1999). The HI was summed over $10^{\prime \prime}$, approximately one beam-width, and contours are $3.75,11.25$, and $18.75 \times 10^{20} \mathrm{~cm}^{-2}$. There could be a small offset in $\mathrm{V}_{\text {sys }}$ between the optical and HI (see Table 3 ).

Fig. 12.- Line-of-sight heliocentric radial velocities $\mathrm{V}_{\text {helio }}$ measured from the stellar absorption lines in each spectrum of NGC 4449. The absorption measurements are steps along the slit summed over $10.65^{\prime \prime}$. The position angle of each spectrum is given; the top left panel is the morphological major axis determined from the V-band image. Position along the slit is measured from the center of the galaxy, seen as the intersection of all the slits in Figure 1, and positive numbers refer to the direction of the given PA. The solid lines are the central velocity. The dashed line is the fit to the rotation velocities of the ionized gas at PA $46^{\circ}$, as shown in Figure 10, for comparison.

Fig. 13. - Spatial representation of the orbits of the gas in the model of NGC 4449: a disk of gas in which tilted orbits precess around the minor axis in an oblate spherical potential. At smaller radii where the precession periods are smaller, the line of nodes regresses from $80^{\circ}\left(\mathrm{r}<2^{\prime}\right)$ to $-110^{\circ}\left(\mathrm{r}>5^{\prime}\right)$, so the gas disk twists. See text for details. Top: The threedimensional model is shown in blue and the projection on the plane of the sky is shown in red. Bottom: The model is shown projected onto the plane of the sky. The solid and dashed blue lines mark the position angles of $46^{\circ}$ and $136^{\circ}$ in the plane of the sky. The line of nodes, shown as the solid black line, is in the plane of the sky in both panels. The two panels are not at the same scale.

Fig. 14. - Model of the gas orbits compared to the observed HI velocities in NGC 4449. The HI position-velocity plots are the same as in Figure 11. The model has been "observed" 
every $15^{\prime \prime}$ along the same position angles as the optical observations. The error bars indicate the velocity "dispersions" due to multiple velocities along the line of sight at those radii. 
This figure "fig1.jpg" is available in "jpg" format from: http://arxiv.org/ps/astro-ph/0207510v1 
This figure "fig2.jpg" is available in "jpg" format from: http://arxiv.org/ps/astro-ph/0207510v1 
This figure "fig3.jpg" is available in "jpg" format from: http://arxiv.org/ps/astro-ph/0207510v1 
This figure "fig4.jpg" is available in "jpg" format from: http://arxiv.org/ps/astro-ph/0207510v1 
This figure "fig5.jpg" is available in "jpg" format from: http://arxiv.org/ps/astro-ph/0207510v1 
This figure "fig6.jpg" is available in "jpg" format from: http://arxiv.org/ps/astro-ph/0207510v1 
This figure "fig7.jpg" is available in "jpg" format from: http://arxiv.org/ps/astro-ph/0207510v1 
This figure "fig8.jpg" is available in "jpg" format from: http://arxiv.org/ps/astro-ph/0207510v1 
This figure "fig9.jpg" is available in "jpg" format from: http://arxiv.org/ps/astro-ph/0207510v1 
This figure "fig10.jpg" is available in "jpg" format from: http://arxiv.org/ps/astro-ph/0207510v1 
This figure "fig11.jpg" is available in "jpg" format from: http://arxiv.org/ps/astro-ph/0207510v1 
This figure "fig12.jpg" is available in "jpg" format from: http://arxiv.org/ps/astro-ph/0207510v1 
This figure "fig13.jpg" is available in "jpg" format from: http://arxiv.org/ps/astro-ph/0207510v1 
This figure "fig14.jpg" is available in "jpg" format from: http://arxiv.org/ps/astro-ph/0207510v1 